\title{
Structure and Chemical Characteristics of Dehydro-L-Ascorbic Acid in Solutions
}

\author{
Yoko NishIKAWA and Tadao KURATA \\ Institute of Environmental Science for Human Life, Ochanomizu University, 2-1-1 Otsuka, Bunkyo-ku, Tokyo 112, Japan
}

Received October 20, 1997; Accepted February 2, 1998

\begin{abstract}
L-Ascorbic acid (ASA) plays an important role in food and biological systems as an electron donor, and in the electron-donating process, ASA itself is generally oxidized to dehydro-L-ascorbic acid (DHA). The structure of DHA was reconfirmed to be monohydrated bicyclic structure in an aqueous solution. It was also clarified that DHA had the solvated bicyclic structure in methanol and ethanol solutions, and in these cases, the enantiomers were formed by the solvation of the $\mathrm{C} 2$ carbonyl group. When these solvated bicyclic DHA were dissolved in water, the solvent molecule on the solvated $\mathrm{C} 2$ carbonyl group was very easily replaced with a water molecule. From the results of MOPAC calculations, the heat of formation of C2 hydrated bicyclic DHA was estimated to be $-299.2 \mathrm{kcal} / \mathrm{mol}$, and DHA was clarified to be considerably stabilized by hydration. Furthermore, it was indicated that bicyclic DHA had more compact and less polar structure than ASA.
\end{abstract}

Keywords: L-ascorbic acid, dehydro-L-ascorbic acid, NMR, MOPAC

In general, L-ascorbic acid (ASA) serves as an electron donor in vivo, for example, in the biosynthesis of collagen (Blanck \& Peterkofsky, 1975), metabolism of xenobiotics (Kato et al., 1977), and scavenging free radicals. In these reactions, ASA becomes monodehydro-L-ascorbic acid (MDA) by the one electron oxidation process (Yamazaki et al., 1960), and successively, dehydro-L-ascorbic acid (DHA). DHA which is considered to be mostly produced from MDA by the disproportionation reaction (Weis et al., 1968) can be easily reduced to ASA by the enzyme, dehydroASA reductase, in vivo. Thus, the biological function of DHA is considered to be very similar to that of ASA because of the easy enzymatic reconversion of DHA to ASA. However, there are some distinct differences between the biological behaviors of ASA and those of DHA which are considered to be mainly due to their structural differences. For example, the transport mechanism of DHA into cells through the cell membrane is thought to be quite different from that of ASA, as is observed in the case of erythrocytes (Hornig et al., 1971), neutrophils (Washko, 1993), epithelial cells of the small intestine (Rose et al., 1988b), and other tissues (Evans et al., 1982; DeChatelet et al., 1974). On the other hand, it was recently suggested that DHA would be one of the important precursors of the Maillard reaction, the so-called aminocarbonyl reaction, in vivo (Ortwerth et al., 1992), which has been suggested to be closely related to aging and the development of age-related diseases such as diabetes (Kornig et al. 1977).

Hitherto, the details of the biological function and also the participation mechanism of DHA in the Maillard reaction in vivo have not been fully clarified, because of a lack of basic chemical and structural information about DHA. For example, most of the DHA preparations used in these studies on the elucidation of the biological function of DHA were obtained by the oxidation of ASA with oxygen gas in the presence of active carbon in methanol $(\mathrm{MeOH})$ or ethanol (EtOH) (Ohmori \& Takagi, 1978), and these preparations have been known to contain $\mathrm{MeOH}$ or EtOH complex whose structure has never been clarified. In this study, in order to clarify these points to some extent, some spectroscopic measurements and also semi-empirical molecular orbital (MO) calculations were made to obtain more detailed information about the structure and chemical characteristics of DHA in solutions.

\section{Materials and Methods}

Reagents used in this research were mostly guaranteed grade reagents of Wako Pure Chemical Industries, Co., Ltd. (Tokyo). Some reagents and deuterium-labeled solvents used in ${ }^{1} \mathrm{H}$ - NMR and ${ }^{13} \mathrm{C}$-NMR measurements were all guaranteed grade reagents of Merck, Inc. ${ }^{1} \mathrm{H}-\mathrm{NMR}$ and ${ }^{13} \mathrm{C}-\mathrm{NMR}$ spectra were obtained by JEOL GSX 270 and JEOL GX 400 FT NMR spectrometers. FAB-mass spectra were recorded with JEOL JMS-700, using glycerol as a matrix and ionization by $\mathrm{FAB}$ with $\mathrm{Xe}$ atoms. Semi-empirical molecular orbital calculations were made by MOPAC (version 6.0), a kind of packaged program for the semi-empirical molecular orbital method (Stewart, 1989a, b). The COMTEC-4DRPC (DAIKIN Co., Ltd., Tokyo) computer and MOLGRAPH (DAIKIN Co., Ltd.) were also used to visualize most of the results of the calculations.

Preparation of $\mathrm{DHA}$ and its derivatives Preparation of DHA was carried out by the method described in the literature (Ohmori \& Takagi, 1978), and DHA was obtained as a pale syrup which was kept in a freezer at $-20^{\circ} \mathrm{C}$ before use. Acetylation of DHA was made as follows: DHA syrup $(1.0 \mathrm{~g})$ was completely dried in a vacuum desiccator, and the dried DHA was dissolved in $6 \mathrm{ml}$ pyridine and added to $6 \mathrm{ml}$ acetic anhydride while stirring and cooling in an ice water bath. The reaction mixture was kept in a refrigerator over- 
night. To this ice water-cooled reaction mixture was added 20 $\mathrm{ml}$ of cold water, and then acetylated DHA was extracted with $30 \mathrm{ml}$ of ether. DHA diacetate was obtained as colorless and transparent crystals by recrystallization from ether, and its structure was confirmed by IR and NMR analyses. Bicyclic ASA derivatives were also prepared using the method described in the literature (Goshima et al, 1973), and the two major products were isolated by preparative TLC and found to be the enantiomers of bicyclic 3-OMe ASA derivatives by NMR analysis.

\section{Results and Discussion}

The structure of DHA which was produced immediately after the oxidation of ASA [1] is usually considered to have a tri-carbonyl- $\gamma$-lactone ring with an extended glycol side chain [2]. The heat of formation of DHA of this nonhydrated form was calculated to be $-223.2 \mathrm{kcal} / \mathrm{mol}$ by
MOPAC. In aqueous solution, however, the hydration of the C2 carbonyl group which is flanked by two other carbonyl groups seems to occur very easily, as is observed in the case of alloxan-hydrate or ninhydrin-hydrate. Although no direct chemical or spectroscopic evidences has yet been obtained for the existence of the $\mathrm{C} 2$ hydrated DHA with an extended side chain [3] in an aqueous solution, the fact that an aqueous solution of freshly prepared DHA shows no strong absorp-<smiles>O=C1OC(C(O)C(O)O)C(O)=C1O</smiles>

H.F. $=-238.6 \mathrm{kcal} / \mathrm{mol}$

ASA

[1]

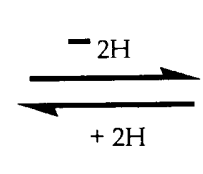

H.F. $=-223.2 \mathrm{kcal} / \mathrm{mol}$ DHA [ 2 ]

\begin{tabular}{lcccccc} 
Table 1. & ${ }^{13} \mathrm{C}$ NMR data for $\mathrm{ASA}$ and DHA. \\
\hline Compound & $\mathrm{C} 1$ & $\mathrm{C} 2$ & $\mathrm{C} 3$ & $\mathrm{C} 4$ & $\mathrm{C} 5$ & $\mathrm{C} 6$ \\
\hline $\mathrm{ASA} / \mathrm{D}_{2} \mathrm{O}$ & 173.8 & 118.0 & 155.6 & 76.3 & 69.0 & 62.2 \\
$\mathrm{DHA} / \mathrm{D}_{2} \mathrm{O}$ & 173.5 & 91.2 & 105.5 & 87.5 & 72.8 & 76.1 \\
$\mathrm{DHA} / \mathrm{CD}_{3} \mathrm{OD}$ & 172.4 & 95.2 & 107.3 & 89.5 & 74.7 & 77.1 \\
& 171.5 & 94.6 & 106.8 & 89.1 & 74.6 & 77.0 \\
\hline
\end{tabular}

(chemical shift $(\delta)$ : ppm)

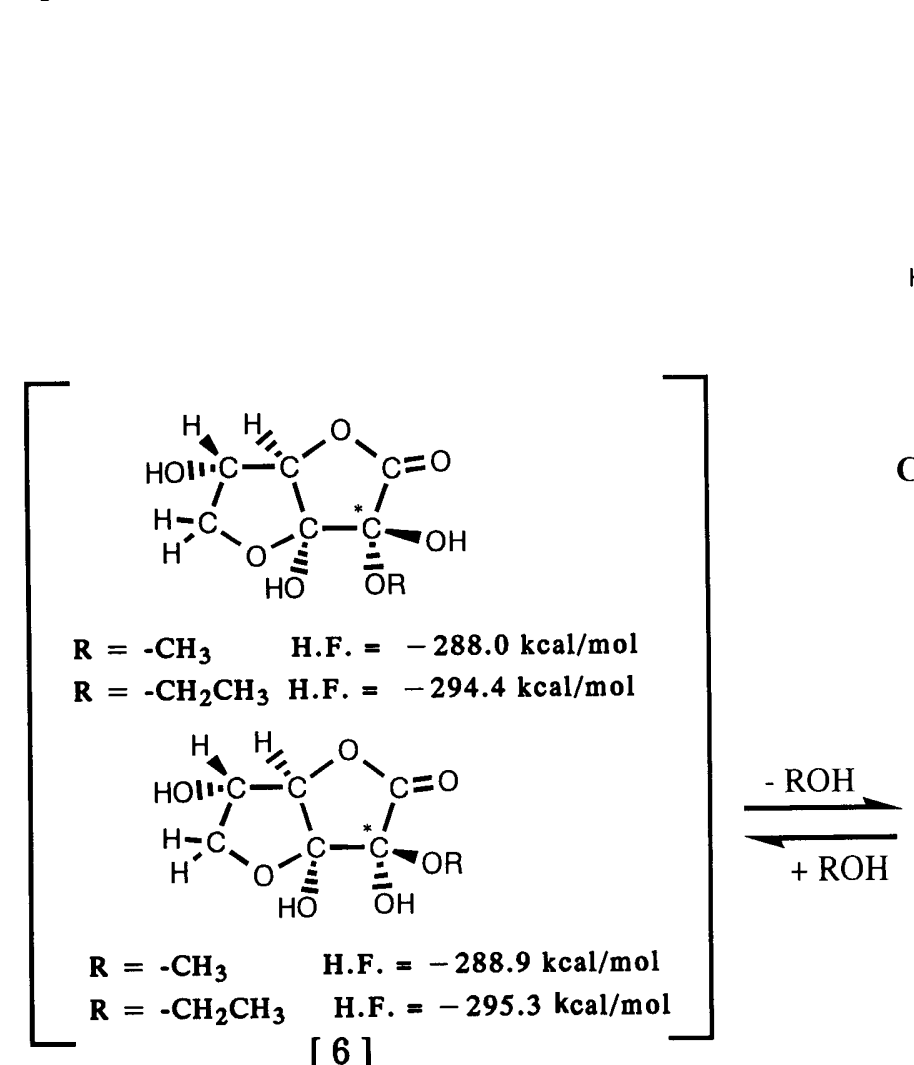

\section{H.F. $=-291.6 \mathrm{kcal} / \mathrm{mol}$ \\ C2 hydrated DHA}

[ 3 ]

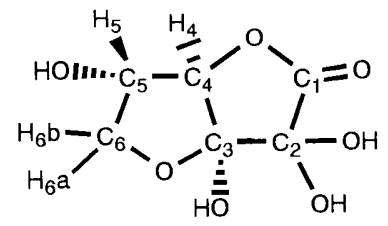

H.F. $=-299.2 \mathrm{kcal} / \mathrm{mol}$

C2 hydrated bicyclic DHA

[4]
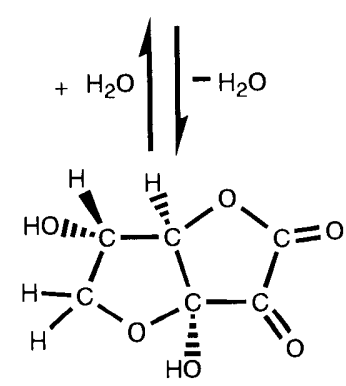

\section{H.F. $=-229.4 \mathrm{kcal} / \mathrm{mol}$ bicyclic DHA}

[5]

Fig. 1. Formation of $\mathrm{C}-2$ solvated DHA from ASA in solution. H.F., heat of formation; *, asymmetric carbon atom which is responsible for the methanol or ethanol complex of DHA. 
tion band in its UV region (above $220 \mathrm{~nm}$ ) seems to indicate the hydration of the carbonyl group. The heat of formation of C2 hydrated DHA [3] was $-291.6 \mathrm{kcal} / \mathrm{mol}$ which suggested the hydrated molecule to be considerably stabilized by the solvation.

On the other hand, the existence of $\mathrm{C} 2$ hydrated bicyclic DHA [4] is easily detected by NMR. The typical ${ }^{1} \mathrm{H}-\mathrm{NMR}$ spectrum of DHA in $\mathrm{D}_{2} \mathrm{O}\left(\delta_{\mathrm{H} 4}=4.76, \delta_{\mathrm{H} 5}=4.36, \delta_{\mathrm{H} 6 \mathrm{a}}=4.28\right.$, $\delta_{\mathrm{H} 6 \mathrm{~b}}=4.16 \mathrm{ppm}, \quad{ }^{3} J_{\mathrm{H} 4 \mathrm{H} 5}=0.7, \quad{ }^{3} J_{\mathrm{H} 5 \mathrm{H} 6 \mathrm{a}}=5.2, \quad{ }^{3} J_{\mathrm{H} 5 \mathrm{H} 6 \mathrm{~b}}=2.7$, ${ }^{2} J_{\mathrm{H} 6 \mathrm{aH} 6 \mathrm{~b}}=10.3 \mathrm{~Hz}$ ) clearly shows a rather simple and characteristic signal pattern due to protons on the hemiketal ring of C2 hydrated bicyclic DHA which was quite identical with the one reported in the literature (Tolbert \& Ward, 1982). The heat of formations of this C2 hydrated bicyclic DHA [4] and non-hydrated one [5] were -299.2 and $-229.4 \mathrm{kcal} / \mathrm{mol}$, respectively, and some stabilization of the bicyclic structures was observed as compared with that of the corresponding monocyclic ones ([3] and [2]).

However, when the spectrum of DHA prepared in $\mathrm{MeOH}$ was observed in $\mathrm{CD}_{3} \mathrm{OD}$, it gave a somewhat similar but more complex signal pattern than that of typical bicyclic DHA in $\mathrm{D}_{2} \mathrm{O}$. This result suggests that the $\mathrm{DHA}$ preparation in $\mathrm{MeOH}$ contains two different molecular species with very similar structures. Also, in the presence of $\mathrm{H}_{2} \mathrm{O}$, this complex signal pattern was changed to a pattern similar to that of typical bicyclic DHA, which seemed to indicate the replacement of the solvated $\mathrm{MeOH}$ on the $\mathrm{C} 2$ carbonyl group with the $\mathrm{H}_{2} \mathrm{O}$ molecule. In fact, the spectrum of DHA in $\mathrm{D}_{2} \mathrm{O}$ showed a singlet due to $\mathrm{MeOH}$, whose signal intensity was confirmed to correspond to three protons. Thus, the liberation of one molecule of $\mathrm{MeOH}$ from one molecule of bicyclic DHA was strongly indicated. The possible existence of two similar species is further supported by the results of ${ }^{13} \mathrm{C}-\mathrm{NMR}$ analysis which show that all carbon signals are observed in pairs, as shown in Table 1. All these results strongly suggested that the two similar molecular species in DHA preparation observed in its NMR spectrum in $\mathrm{CD}_{3} \mathrm{OD}$ were enantiomers of $\mathrm{C} 2$ solvated bicyclic DHA as illustrated in Fig. 1 [6].
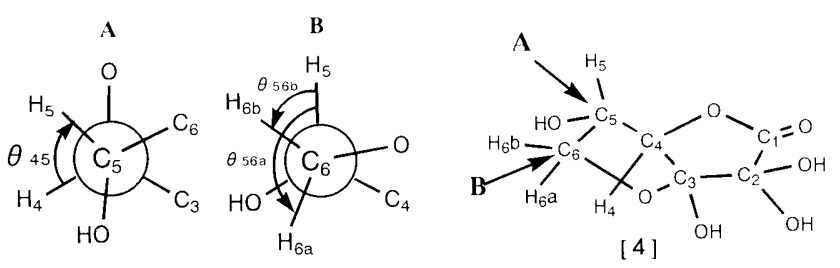

\begin{tabular}{|c|c|c|c|}
\hline \multirow{2}{*}{$\begin{array}{c}\text { Definition of } \\
\text { tortion angle }\end{array}$} & ${ }^{3} \mathrm{~J}_{\mathrm{HH}}(\mathrm{Hz})$ & \multicolumn{2}{|c|}{ Tortion angle (degrec) } \\
\cline { 3 - 4 } & 0.7 & 101.9 & 118.2 \\
\hline 1. H4-C4-C5-H5 & 5.2 & 130.7 & 128.0 \\
\hline 2. H5-C5-C6-H6a & 2.7 & 55.6 & 4.2 \\
\hline 3. H5-C5-C6-H6b & & KOPAC $^{* *}$ \\
\hline
\end{tabular}

Fig. 2. The side-chain structure of DHA in an aqueous solution. *These torsion angles were calculated from the coupling constant by the Karplus equation $\left({ }^{3} J_{\mathrm{HH}}=10.5 \cos ^{2} \theta-1.2 \cos \theta\right)$. ${ }^{* *}$ These torsion angles were obtained from the optimized structure of DHA calculated by MOPAC.
Although it has long been known that the synthesis of DHA in $\mathrm{MeOH}$ produces $\mathrm{MeOH}$ complexes of $\mathrm{DHA}$, the structure of the complex has never been clarified. However, from our results of NMR analyses, the $\mathrm{MeOH}$ complex of DHA was confirmed to be the enantiomers of $\mathrm{C} 2$ solvated bicyclic DHA which was further supported by the results of FAB-MS analysis. The FAB-mass spectrum of DHA synthesized in $\mathrm{MeOH}$ showed the presence of the DHA adduct with methanol $\left([\mathrm{M}+\mathrm{H}]^{+}, m / z 207\right)$ as one of the major peaks. Two other peaks $m / z 175\left([\mathrm{M}+\mathrm{H}-\mathrm{MeOH}]^{+}\right)$and $m / z 189$ $\left(\left[\mathrm{M}+\mathrm{H}-\mathrm{H}_{2} \mathrm{O}\right]^{+}\right)$, thought to be derived from the DHA adduct with $\mathrm{MeOH}(\mathrm{m} / \mathrm{z} 207)$, were also observed in rather high relative abundance. In the higher mass region, peaks of the DHA adduct with matrix glycerol $(m / z 267), \mathrm{H}_{2} \mathrm{O}$ glycerol $(m / z 285)$ and $\mathrm{MeOH}$-glycerol $(\mathrm{m} / z$ 299) were also observed. Furthermore, the ${ }^{1} \mathrm{H}-\mathrm{NMR}$ spectrum of acetylated DHA showed two methyl signals $(\delta=2.19,2.13 \mathrm{ppm})$ corresponding to two acetyl groups (data not shown) which indicated that two hydroxy groups $(\mathrm{C} 3-\mathrm{OH}$ and $\mathrm{C} 5-\mathrm{OH})$ existed in $\mathrm{C} 2$ solvated bicyclic DHA molecule. Also, the ${ }^{1} \mathrm{H}-\mathrm{NMR}$ spectrum of the bicyclic derivatives of 3-OMe ASA showed two singlets $(\delta=3.42,3.49 \mathrm{ppm})$ due to the $3-\mathrm{OMe}$ group of two enantiomers, while DHA preparation gave no methyl signals around $3.4 \mathrm{ppm}$ suggesting the location of the $\mathrm{MeOH}$ molecule on the $\mathrm{C} 2$ carbonyl group of DHA by solvation.

The signal pattern shown in the ${ }^{1} \mathrm{H}-\mathrm{NMR}$ spectrum (in $\mathrm{CD}_{3} \mathrm{OD}$ ) of the DHA sample prepared by the oxidation of ASA in absolute EtOH was more complicated than that of C2 $\mathrm{MeOH}$ solvated bicyclic DHA (data not shown). This result also suggested the presence of enantiomers of $\mathrm{C} 2 \mathrm{EtOH}$ solvated bicyclic DHA. In the presence of $\mathrm{H}_{2} \mathrm{O}$, the complex signal pattern was easily changed to a more simple one which was the same signal pattern as the one in $\mathrm{D}_{2} \mathrm{O}$.

All these facts suggested that $\mathrm{H}_{2} \mathrm{O}$ molecules were able to bind to the $\mathrm{C} 2$ carbonyl group more strongly than $\mathrm{MeOH}$ and EtOH molecules. This phenomenon was further substantiated by the results of MOPAC calculations which showed that the heat of formation of C2 hydrated bicyclic DHA ( -299.2 $\mathrm{kcal} / \mathrm{mol}$ ) was lower than the value of the heat of formation for the corresponding $\mathrm{MeOH}$-solvated bicyclic DHA (ca -288 to $-289 \mathrm{kcal} / \mathrm{mol}$ ) and that for the ethanol-solvated one (ca. -294 to $-295 \mathrm{kcal} / \mathrm{mol}$ ) as shown in Fig. 1 [6].

To obtain more detailed structural information about the side chain of DHA in aqueous solution, Karplus equation and MOPAC calculation were employed to evaluate some torsion angles of the side chain of DHA as shown in Fig. 2. The calculations using the Karplus equation were based on the spin-spin coupling constant of DHA observed in the

Table 2. Some physicochemical parameters of ASA and non-hydrated bicyclic DHA calculated by MOPAC.

\begin{tabular}{lcc}
\hline Physicochemical parameters & ASA & Non-hydrated bicyclic DHA \\
\hline Molecular volume & $140.8 \AA^{3}$ & $131.3 \AA^{3}$ \\
Molecular length $^{a)}$ & $7.43 \AA$ & $6.25 \AA$ \\
Dipole moment & $3.20 \mathrm{D}$ & $2.71 \mathrm{D}$ \\
\hline
\end{tabular}

a) Molecular length was tentatively defined to be equal to the greatest value of interatomic distances. 
${ }^{1} \mathrm{H}-\mathrm{NMR}$ spectrum taken in $\mathrm{D}_{2} \mathrm{O}$. The values of the torsion angle of H5-C5-C6-H6a calculated by the Karplus equation and MOPAC were very similar, while those of H4-C4-C5-H5 were a little different and those of $\mathrm{H} 5-\mathrm{C} 5-\mathrm{C} 6-\mathrm{H} 6 \mathrm{~b}$ were significantly different. The disagreements in the calculated values of these torsion angles might be ascribed the fact that MOPAC calculations were made on a completely isolated molecule neglecting the possible various solvent effects.

As mentioned earlier in this report, the difference between the transport mechanism of ASA and that of DHA into cells is considered to be basically ascribable to their structural differences. The chemical structure of DHA in aqueous solution has been demonstrated to be the bicyclic structure from the results of ${ }^{1} \mathrm{H}-\mathrm{NMR}$ and ${ }^{13} \mathrm{C}-\mathrm{NMR}$ analyses as previously described. Therefore, a comparison of the volumes, the lengths and dipole moments of the molecules of ASA and non-hydrated bicyclic DHA obtained by MOPAC calculation was made, as there had been no previous report which described such a comparison on these molecules. Table 2 summarizes the results of the calculations which show that the bicyclic structure of DHA was rather compact and less polar than ASA. Evidently, these differences in their physicochemical characteristics such as dipole moment and molecular size will have some effects on their biological behavior, for example, the transportation mechanism through the biomembrane. Therefore, bicyclic DHA which has more compact and less polar structure than ASA will be able to more easily penetrate into the relatively non-polar hydrophobic region of the membrane than ASA. This may partly explain the difference in the transportation mechanisms of ASA and DHA reported earlier (Rose, 1988a). Further studies are necessary to obtain more precise and conclusive information about the physicochemical behavior of DHA molecules in solution.

\section{References}

Blanck, T.J.J. and Peterkofsky, B. (1975). Stimulation of collagen secretion by ascorbate as a result of increased proline hydroxylation in chick embryo fibroblasts. Arch. Biochem. Biophys., 171, 259-267.
DeChatelet, L.R., McCall, C.E., Cooper, M.R. and Shirley, P.M (1974). Ascorbic acid levels in phagocytic cells. Proc. Soc. Exp. Biol. Med., 145, 1170-1173.

Evans, R.M., Currie, L. and Campbell, A. (1982). The distribution of ascorbic acid between various cellular components of blood, in normal individuals, and its relation to the plasma concentration. $B r$. J. Nutr., 47, 473-482.

Goshima, K., Maezono, N. and Tokuyama, K. (1973). A novel degradation pathway of L-ascorbic acid under non-oxidative conditions. Bull. Chem. Soc., 46, 902-904.

Hornig, D., Weiser, H., Weber, F. and Wiss, O. (1971). Uptake and release of [1-14C]-ascorbic acid and [1-14C]-dehydroascorbic acid by erythrocytes of guinea pigs. Clin. Chim. Acta, 32, 33-39.

Kato, N., Okada, T., Takenaka, Y. and Yoshida, A. (1977). Ameliorative effect of dietary ascorbic acid on PCB toxicity in guinea pigs. Nutr. Rep. Int., 15, 125-130.

Kornig, R.J., Blobstein, S.H. and Cerami, A. (1977). Structure of carbohydrate of hemoglobin $A_{1 C}$. J. Biol. Chem., 253, 2992-2997.

Ohmori, M. and Takagi, M. (1978). A facile preparation of dehydroascorbic acid-methanol Solution and its stability. Agric. Biol. Chem., 42, 173-174.

Ortwerth, B.J., Slight, S.H., Prabhakaram, M., Sun, Y. and Smith, J.B. (1992). Site-specific glycation of lens crystallins by ascorbic acid. Biochem. Biophys. Acta, 1117, 207-215.

Rose, R.C. (1988a). Transport of ascorbic acid and other water-soluble vitamins. Biochim. Biophys. Acta, 947, 335-366.

Rose, R.C., Choi, J.L. and Koch, M.J. (1988b). Intestinal transport and metabolism of oxidized ascorbic acid (dehydroascorbic acid). Am. J. Physiol., 254, G824-G828.

Shukla, A., Dikshi, M. and Srimal, R. (1995). Status of antioxidants in brain microvessels of monkey and rat. Free Rad. Res., 22, 303-308.

Stewart, J.J.P. (1989a). Optimization of parameters for semiempirical methods. I. Methods. J. Comp. Chem., 10, 209-220.

Stewart, J.J.P. (1989b). Optimization of parameters for semiempirical methods. II. Applications. J. Comp. Chem., 10, 221-264.

Tolbert, B.M. and Ward, J.B. (1982). "Ascorbic acid: Chemistry, Metabolism, and Uses," ed. by P.A. Seib and B.M. Tolbert Am. Chem. Soc., Washington, pp. 101-123.

Washko, P.W., Wang, Y. and Levine, M. (1993). Ascorbic acid recycling in human neutrophils. J. Biol. Chem., 268, 15531-15535.

Weis, W., Zubrzycki, Z. and Staudinger, H. (1968). Redox behavior of $\mathrm{L}(+)$-semidehydro-ascorbic acid. Hoppe-Seyler's Z. Physiol. Chem., 349, 279-282.

Yamazaki, I., Mason, H.S. and Piettem, L.H. (1960). Identification by electron paramagnetic resonance spectroscopy of free radicals generated from substrates by peroxidase. J. Biol. Chem., 235, 24442449 . 